\title{
DIFFUSE COPLANAR SURFACE BARRIER DISCHARGE IN NITROGEN: MICRODISCHARGES STATISTICAL BEHAVIOR
}

\author{
Jan Cech*, Jana Hanusova, Pavel Stahel, Pavel Slavicek \\ Masaryk University, Regional REDD center for low-cost plasma and nanotechnology surface modifications, \\ Faculty of Science, Masaryk university, Kotlarska 2, 61137 Brno, Czech Republic \\ * corresponding author: cech@physics.muni.cz
}

\begin{abstract}
We studied statistical behavior of microdischarges of diffuse coplanar surface barrier discharge (DCSBD) operated in nitrogen atmosphere at two input voltage regimes. We measured spectrally unresolved discharge patterns together with discharge electrical parameters using highspeed iCCD camera and digital storage oscilloscope. External synchronization enabled us to measure the discharge pattern during positive and/or negative half-period of input high voltage in the single-shot mode of operation. The comparison of microdischarges behavior during positive, negative and both half periods of input high voltage was performed for two levels of input voltage, i.e. voltage slightly above ignition voltage and high above ignition voltage ("overvoltage"). The number of microchannels crossing discharge gap was counted and compared with number of microdischarge current peaks observed during corresponding half-period of input high voltage. The relations of those incidences was shown and discussed.
\end{abstract}

KEYwORDS: DCSBD, diffuse coplanar surface barrier discharge, microdischarges, time resolved imaging, iCCD.

\section{INTRODUCTION}

In past decades the importance of barrier discharges as the sources of non-equilibrium plasmas for material processing has raised [3. As a type of planar configuration of barrier discharge so called diffuse coplanar surface barrier discharge (DCSBD) was invented by prof. Cernak [1].

Plasma of DCSBD is generated in thin layer above dielectric, at relatively high power densities of the order of $100 \mathrm{~W} \mathrm{~m}^{-3}$. The discharge consists of thin channels (filaments or micro-discharges) crossing the electrode gap between electrodes [2] and visually diffuselike layer above electrodes. These properties make DCSBD a promising candidate for high-speed plasma processing of various materials [1].

Because of its application potential coplanar barrier discharge properties and potential for applications have been investigated in recent decade [4, 5].

In presented paper the statistical behavior of DCSBD microdischarges operated in nitrogen was studied by means of high-speed iCCD camera imaging synchronized with the power supply generator and digital storage oscilloscope. The relation of number of microfilament channels crossing discharge gap and number of current peaks observed during corresponding half-period of input high voltage was studied.

\section{EXPERIMENTAL SETUP}

For presented study the simplified DCSBD cell or system was used. The one-electrode-pair discharge cell was used. In Fig. 1 (left) the cross-section of discharge cell is given. The discharge cell is made of polymer capsule in which the system of semi-movable electrodes is placed. Both electrodes are pressed against dielectric plate and dipped in insulating oil bath. The arbitrary rectangular electrode gap between electrodes can be set with width up to $5 \mathrm{~mm}$. The minimum distance between electrodes is governed by the insulating properties of oil bath. In presented study the electrode distance was set to $0.55 \pm 0.05 \mathrm{~mm}$.

The dielectric plate is pressed directly to the surface of electrodes, which form two semicircle footprints on the dielectric plate, forming rectangular electrode gap in between. The schematic view of the electrodes groundplan is given in Fig. 1 (right). The view plane of the picture is the same as if the paper would be the dielectric plate. The diameter of semicircle electrodes is approx. $20 \mathrm{~mm}$.

Discharge chamber is placed from the opposite side of dielectric plate; see Fig. 1 (left). The dielectric plate is made of $96 \%$ alumina $\left(\mathrm{Al}_{2} \mathrm{O}_{3}\right)$ with dimensions of $10 \times 10 \mathrm{~cm}$ and thickness of approximately $0.6 \mathrm{~mm}$. Discharge chamber enables us to operate the discharge in controlled working gas environment. Quartz window on the opposite side of discharge chamber enables us to perform optical diagnostics of the discharge.

Experimental setup for time resolved iCCD measurements is given in Fig. 2 The discharge cell described in previous section was used.

Voghtlin Instruments red-y GCR gas flow controller was used to set requested working gas atmosphere. Nitrogen gas of the purity better than $99.996 \%$ and total gas flow rate of $3 \mathrm{slpm}$ was used. 


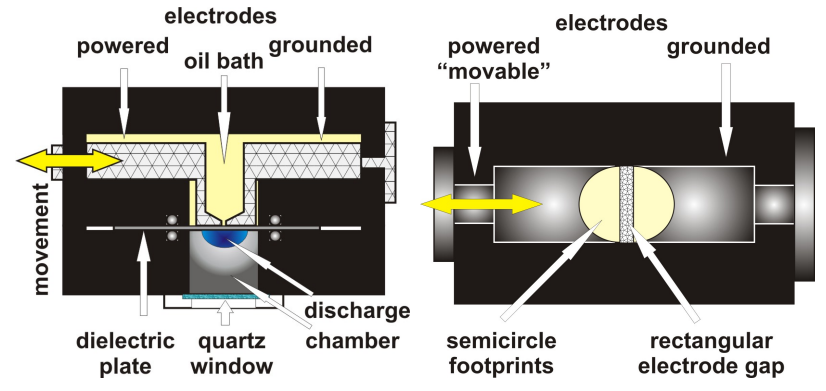

FiguRE 1. Experimental setup II: a) discharge chamber cross-section, b) electrode system groundplan.

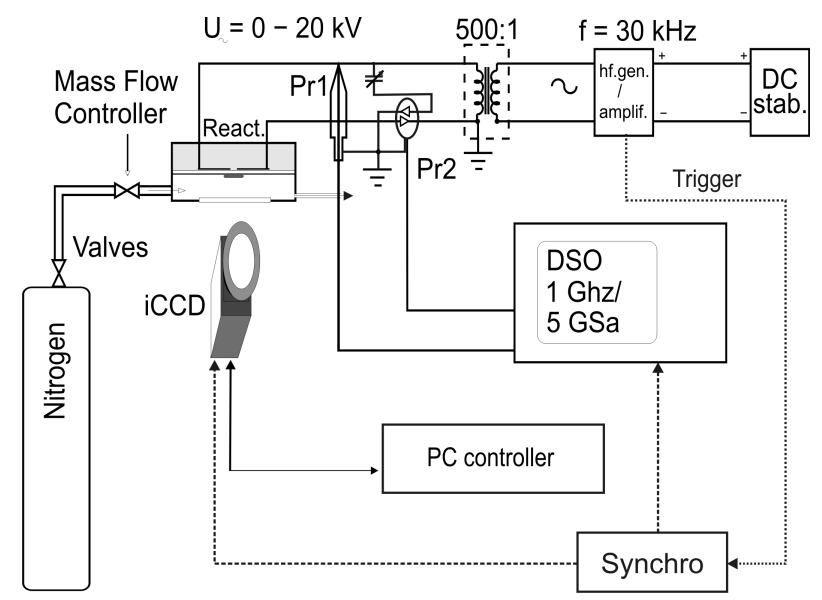

FiguRE 2. Experimental setup: general scheme of discharge setup and time-resolved optical imaging.

High voltage (HV) power supply was used to ignite and maintain the discharge. The $\mathrm{HV}$ power supply consists of high frequency tunable generator LIFETECH HF Power Source powered by stabilized DC power source STATRON 3262 and LIFETECH $\mathrm{HV}$ transformer. The HV power supply was operated at $30 \mathrm{kHz}$ and $24 \mathrm{kV}$ peak-to-peak, resp. $32 \mathrm{kV}$ peakto-peak, sine-wave. These voltage levels correspond to the level of "just ignited" discharge and discharge operated at high "overvoltage" level above ignition voltage.

The current-voltage characteristics were recorded using LeCroy WaveRunner 6100A 1 GHz/5 GSa digital storage oscilloscope coupled with HV probe Tectronix P6015A 1000:1 (in Fig. 2 Denoted as Pr1) and Pearson Current Monitor 2877 (in Fig. 2 denoted as Pr2). Variable high voltage capacitor was used as the displacement current compensator. The HV capacitor was connected antiparallel through current probe. Tuning the HV capacitor to the capacity close to discharge cell capacity effectively reduces measured displacement current of discharge cell which is of the same order as the discharge current. This increases effectively the signal-to-noise ratio of discharge current measurements.

For the high speed synchronized discharge imaging, the Princeton Instruments PI-MAX 1024RB-25-FG43 iCCD camera equipped with $50 \mathrm{~mm}, \mathrm{f} / 2.8 \mathrm{UV}$ lens was used. The iCCD camera was placed along axis of symmetry perpendicular to DCSBD plasma layer.

To synchronize and semi-automate the measurements, the Agilent 33220A function generator was used as trigger. As the source signal for trigger the reference signal of HF generator was used. The Agilent 33220 A fires triggering signals for synchronous iCCD image capture together with current-voltage measurement of the same event.

This setup enables us to take series of synchronized images of the discharge pattern together with its current-voltage characteristics with the resolution of single half-period of high voltage waveform. In presented work the 100 images series of first, second and both half-periods were taken with gate times of 17 , resp. $34 \mu \mathrm{s}$. The iCCD delays were set in the way to guarantee that images represent all discharge events of half-periods that can be identified in current-voltage waveforms.

\section{Results AND Discussion}

For each half period of the discharge the 100 events were recorded, that gives 600 discharge pattern images and also current-voltage measurement. In the first step of data processing the discharge current corresponding to specified half period of input high voltage was analyzed. The peak detection algorithm was adopted to evaluate the current peaks total number, together with their polarity and amplitude. In the second step of data processing the corresponding iCCD images of discharge pattern were processed. For analysis of discharge microchannels the narrow rectangular region corresponding to inter-electrode rectangular gap (see Figs. 1 and 3) was selected. The total number of microdischarges (bright channels) crossing the electrode gap was obtained together with their positions and amplitudes using similar peak detection algorithm used for discharge current analysis. Finally the data sets of current peaks events and microdischarge channels events were processed to obtain relation frequency matrix of incidence described in next section.

In Fig. 3 the iCCD images of the discharge pattern for input voltage of $24 \mathrm{kV}$ is shown. The central part of images corresponding to electrode gap was processed to evaluate microchannels crossing the gap. Images represent single shots of discharge microchannels incidence during one half period of input high voltage. In Fig. 3 the left, resp. middle image represents positive, resp. negative half period of discharge with respect to the polarity of the discharge electrodes (shown for clarity only in the first image), image on the right represents whole period of discharge. Polarity is taken as polarity of HV signal on the left electrode, see Figs. 1 and 2 .

In Fig. 4 the typical current-voltage characteristics of DCSBD operated in nitrogen is given. The numerous current peaks per half period of input high voltage can be seen. The reference triggering signal used for diagnostics synchronization is also depicted. 

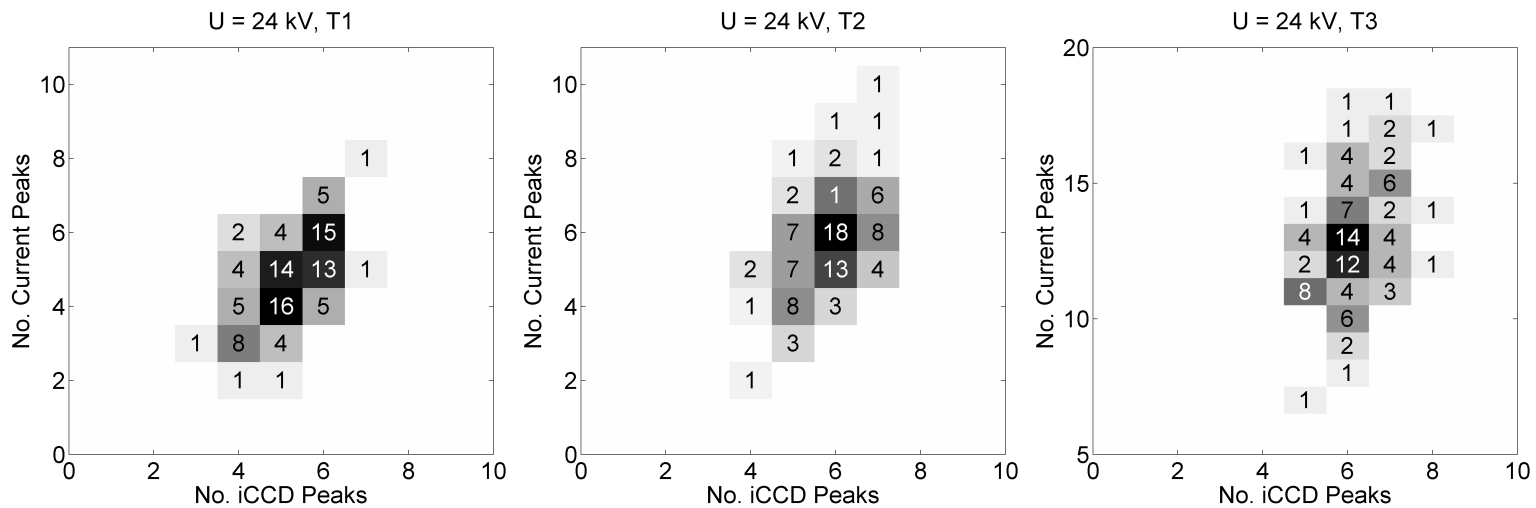

FIGURE 5. Relations of number of iCCD identified microchannels crossing discharge gap and number of current peaks in corresponding half period, input voltage is $24 \mathrm{kV}$ (pk-pk); figures represents: a) positive half period, b) negative half period and c) whole period of input high voltage.
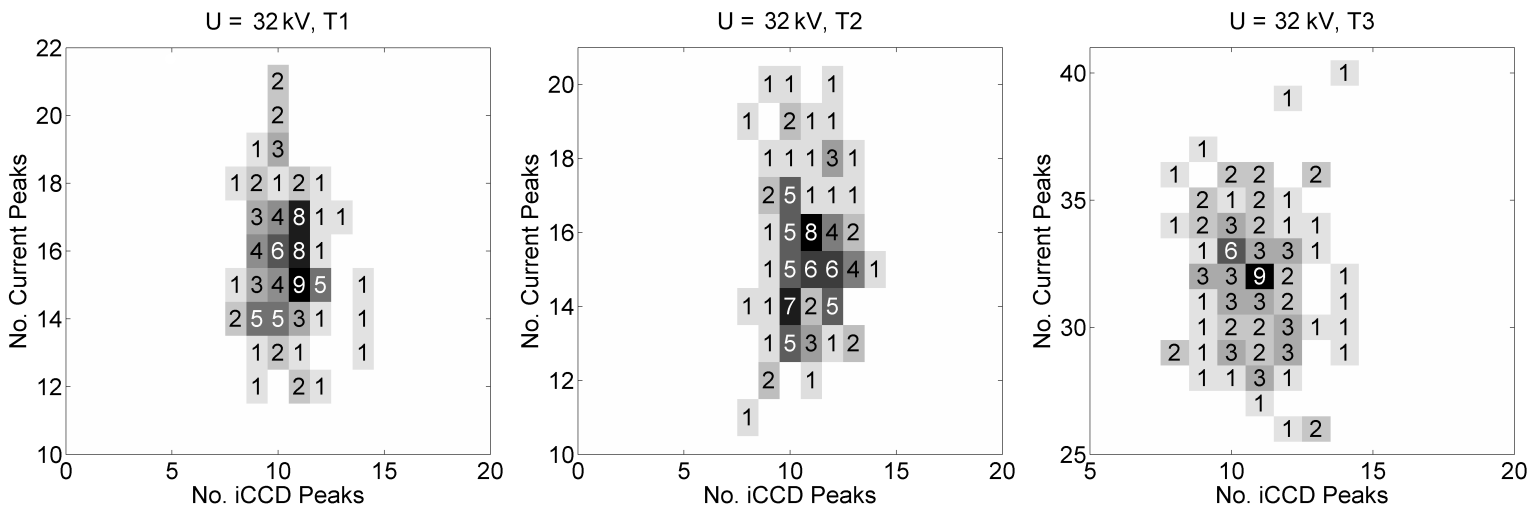

Figure 6. Relations of number of iCCD identified microchannels crossing discharge gap and number of current peaks in corresponding half period, input voltage is $32 \mathrm{kV}$ (pk-pk); figures represents: a) positive half period, b) negative half period and c) whole period of input high voltage.
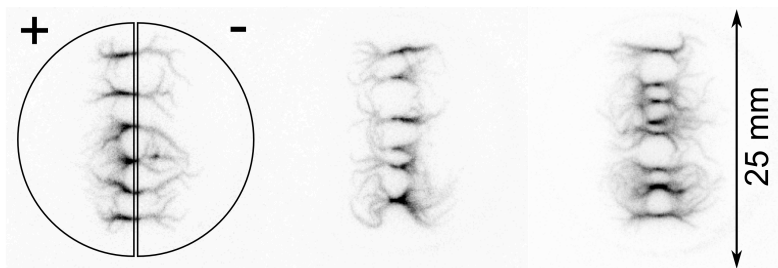

FIGURE 3. Time resolved iCCD images of DCSBD, $d=0.6 \mathrm{~mm}, U_{\mathrm{pk}-\mathrm{pk}}=24 \mathrm{kV}$; half-periods: left to right: positive, negative, both.

In Figs. 5 and 6 the resulting relation frequency matrices of incidence is given. The matrices represent evaluation of microdischarges behavior. On $x$ axis the number of microchannels crossing the gap during one half period (whole period) is given. On $y$ axis the corresponding number of microdischarge current peaks within the same half period (whole period) is given. The numbers in matrices represents numbers of incidences of described events. From the incidence matrices the statistical behavior of the DCSBD microdischarges (of filaments) can be seen. In case of 1:1 correspondence, where each microdischarge will produce unique microchannel crossing the discharge gap, all events will be positioned along main diagonal

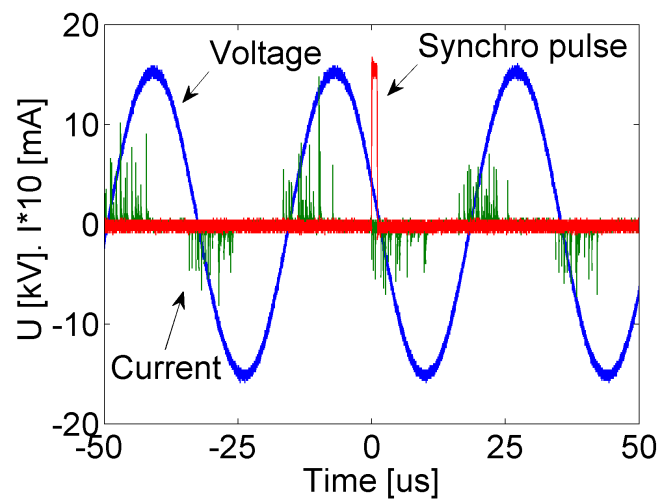

FiguRE 4. Typical current-voltage characteristics of DCSBD operated at $32 \mathrm{kV}$ (pk-pk); synchronization signal used for synchronization of iCCD camera and digital storage oscilloscope is shown.

of the incidence matrix. This tendency can be seen in Fig. 5 for the matrices representing positive (T1) and negative (T2) half period of discharge for input voltage of $24 \mathrm{kV}$. It can be seen, that the substantial part of events is distributed along the main diagonal. In case of whole period (T3) the most occurring event corresponds to case where only half number 
of unique microchannels crossed the discharge gap in comparison of total microdischarges current peaks within the period. Notice the different scale (section) of incidence matrix in case of whole period.

When we compare the behavior of filaments of DCSBD operated at very low input voltage (Fig. 5) with behavior under "overvoltage" of input voltage (Fig. 6), we can see substantial differences. Please notice the shifted scales (sections) of corresponding incidence matrices in Figs. 5 and 6. With agreement to the research performed by Hoder [2] on the single-filament DCSBD configuration, the filaments of DCSBD in the regime of "overvoltage" prefer to prolongate or branch existing microchannels against formation of new microchannels crossing the electrode gap. From the presented data the tendency is that the substantial number of microdischarges (identified as discharge current peaks) do not form new microchannels but rather prolongate or branch existing discharge microchannels.

\section{Conclusion}

In this paper we have presented time resolved optical measurements of DCSBD in nitrogen. With presented experimental setup we were able to take single-shot pictures of discharge pattern with the resolution of half-period of input voltage. The behavior of DCSBD microdischarges in nitrogen was studied for two different input voltage regimes. The incidence frequency matrices of microdischarges and microchannels occurrence were derived. It has been investigated, that under low input voltage conditions, the preferred way of microdischarge behavior is formation of unique microchannel per microdischarge. Contrary in the case of substantially increased input voltage ("overvoltage") the preferred microdischarges behavior is to reuse existing microchannels and/or prolongate or branch existing microchannels instead of forming completely new microchannels. Obtained results support the previous measurements of DCSBD performed by Hoder in single-filament DCSBD configuration [2]. To distinguish between different regimes of microdischarges behavior further investigations has to be carried.

\section{ACKNOWLEDGEMENTS}

This research has been supported by the project Regional R\&D center for low-cost plasma and nanotechnology surface modifications CZ.1.05/2.1.00/03.0086 funded by European Regional Development Fund and projects No. TA01011356/2011 and TA01010948/2011 of the Technology Agency of the Czech Republic.

\section{REFERENCES}

[1] M. Cernak, L. Cernakova, I. Hudec, et al. Diffuse coplanar surface barrier discharge and its applications for in-line processing of low-added-value materials. European Physical Journal-Applied Physics 47(2):22806, 2009.

[2] T. Hoder. Studium filamentu koplanarniho barieroveho vyboje. Masaryk University, Brno, 2009. Ph.D. Thesis.

[3] U. Kogelschatz. Dielectric-barrier discharges: Their history, discharge physics, and industrial applications. Plasma Chemistry and Plasma Processing 23(1):1-46, 2003.

[4] D. Korzec, E. G. Finantu-Dinu, G. L. Dinu, et al. Comparison of coplanar and surface barrier discharges operated in oxygen-nitrogen gas mixtures. Surface and Coatings Technology 174-175:503-508, 2003.

[5] M. Stefecka, M. Kando, M. Cernak, et al. Spatial distribution of surface treatment efficiency in coplanar barrier discharge operated with oxygen-nitrogen gas mixtures. Surface and Coatings Technology 174-175:553-558, 2003. 